\title{
RESTORATION OF THE ATMOSPHERE IN SOLAR MODELS
}

\author{
P. MOREL ${ }^{1}$, C. VAN'T VEER ${ }^{2}$ \\ 1 Cassini, URA CNRS 1362, Observatoire de la Côte d'Azur, Nice \\ 2 DASGAL, URA CNRS 335, Observatoire de Paris, Paris
}

G. BERTHOMIEU ${ }^{1}$, R. CAYREL ${ }^{2}$, F. CASTELLI ${ }^{3}$, M.J. GOUPIL ${ }^{2}$, Y. LEBRETON $^{2}$, J. PROVOST ${ }^{1}$

${ }^{3}$ Observatorio Astronomico, Trieste

\begin{abstract}
From models of the Sun atmosphere obtained with ATLAS 9 (Kurucz 1992) it appears that the diffusion limit of the transfer equation becomes valid only at depths larger than $\tau_{R} \sim 10$. Even with physics as consistent as possible one restores the atmosphere with an accuracy not better than $\sim 40 \%$, but with small consequences on solar calibrated models. Despite the eigenmodes have turning points located in the atmosphere, the study of the oscillations reveals differences less than $2 \mu \mathrm{Hz}$ when the atmosphere is restored with various assumptions.
\end{abstract}

\section{I - OPTICAL DEPTH REQUIRED BY THE DIFFUSION LIMIT}

An accurate description of the atmosphere is needed for precise stellar interior calculations. Since it is out of possibilities to include full atmosphere calculations into a stellar model, one separates the interior where the diffusion approximation is valid from the atmosphere which is restored from a $T(\tau)$ law, the limit between the two parts beeing located at a large Rosseland optical depth $\tau_{b}$.

The so-called diffusion approximation only valid at great optical depths writes (Mihalas, 1978):

$$
\lim _{\tau_{\nu} \rightarrow \infty} H_{\nu}=-\frac{1}{3} \frac{\partial B_{\nu}}{\partial \tau_{\nu}}
$$

If, in some parts of the spectrum such as in spectral lines, this limit is reached at depths that correspond to a few tenths in the Rosseland optical depth $\tau_{R}$, in other parts where the medium is less opaque, it is far to be fullfilled even if $\tau_{R}>1$. Hence, for the calculation of the radiative flux one needs an estimate $\tau_{b}$ of the optical depth above which the value of the integrated radiative flux $H$ reaches its optically thick limit.

Along the solar evolutionary track from the ZAMS to now: the gravity is almost constant and the effective temperature $T_{\text {eff }}$ varies from $\sim 5700^{\circ} \mathrm{K}$ to $\sim 5780^{\circ} \mathrm{K}$. With the code ATLAS 9 (Kurucz, 1992) we have calculated atmosphere models of a star of $1 M_{\odot}$ with $T_{e f f}=5650^{\circ} \mathrm{K}, 5700^{\circ} \mathrm{K}, 5750^{\circ} \mathrm{K}, 5800^{\circ} \mathrm{K}(\log g=4.47, \alpha=1 / \mathrm{Hp}=1.7$, $X=0.70, Y=0.28, Z=0.02$ ) and compared the radiative flux derived from the diffusion approximation to its exact value. From the Fig.I the relative difference of these two fluxes remains greater than $1 \%$ above $\tau_{R}=10$. As a consequence one should set $\tau_{b} \geq 10$, provided the $T(\tau)$ law includes the convective transport. However from table I one sees that the global parameters of the model are not significantly affected by changes of $\eta_{b}$, except for the radiative Eddington's law. 


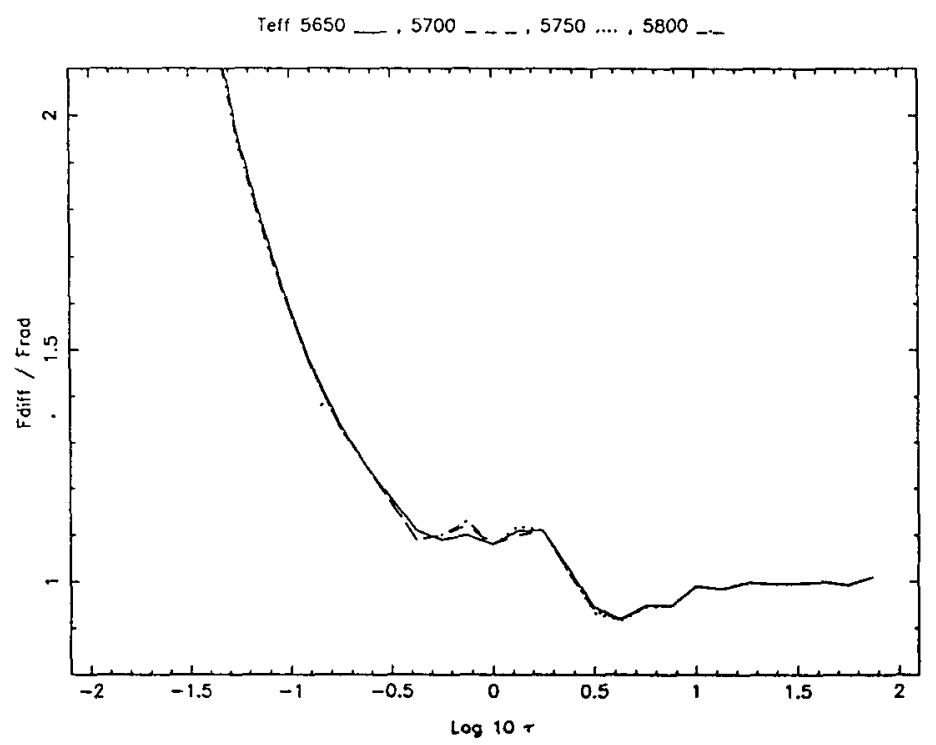

FIGURE I Ratio between the radiative flux and its optically thick value derived by the diffusion equation. The curves are drawn for various $T_{\text {eff }}$. Only for $\tau_{R} \geq 10$ the relative difference is less than $1 \%$.

\section{II - RESTORATION OF AN ATMOSPHERE WITH A $T(\tau)$ LAW}

In stellar evolution calculations the derivation of the temperature needs the knowledge of the radiative and convective fluxes. In the optically thick medium the radiative flux can be derived from the diffusion equation but, for $\tau_{R} \leq 10$, much more sophisticated radiative transfer computations are needed. These heavy calculations can be avoided by the knowledge a priori of the temperature as a function of some parameters of the model. For the Sun one writes the temperature as a function of the effective temperature and of the Rosseland optical depth $T\left(\tau, T_{e f f}\right)$. The $T(\tau)$ laws are derived either from theory (Eddington, Hopf) or from models (HSRA). The temperature dependence with respect to the optical depth is smooth enough so that, within $\pm 0.5 \%$, for any $T_{e f f}$, in the range $5700^{\circ} \mathrm{K} \leq T_{\text {eff }} \leq 5800^{\circ} \mathrm{K}$ the temperature can be restored by a "tangent" Hopf's law:

$$
T=T_{e f f}\left[\frac{3}{4}(\tau+\tilde{q}(\tau))\right]^{1 / 4}, \text { with the function } \tilde{q}(\tau)=\frac{4}{3}\left(\frac{T}{\bar{T}_{\text {eff }}}\right)^{4}-\tau
$$

obtained from a $1 M_{\odot}$ model of atmosphere with $\bar{T}_{\text {ef } f}\left(=5750^{\circ} \mathrm{K}\right.$ hereafter $)$.

Throughout the atmosphere the luminosity is equal to its value at the bottom: $L=L\left(r_{0}\right)=L_{\text {. }}$. The star radius $R_{*}$ is defined as the radius at $\tau_{*}$ where $T=T_{\text {eff }}=$ $\left(L_{*} / 4 \pi \sigma R_{*}^{2}\right)^{1 / 4}$ which reduces to $\tau_{*}=2 / 3$ with the Eddington law. The set of equation to be solved can be found in Morel et al. (1990).

The degree of accuracy of the restored atmosphere can be assessed by comparing the values of physical quantities such as pressure, temperature and sound speed as provided by the full atmosphere model calculation and the restored atmosphere. Even if the restoration is made with the maximum of consistency, as we attempted, with respect to the geometrical depth the pressure (respt. the sound speed) is hardly restored with an accuracy better than $40 \%$ (respt. $5 \%$ ). 


\section{III - RESULTS AND DISCUSSION}

A set of calibrated solar models with different $T(\tau)$ laws and different values of $\tau_{\star}$ and $\tau_{b}$ and their p modes oscillations frequencies have been computed, using the Nice CESAM code (these proceedings). Their global properties are given in Table I.

The model SS is obtained with a boundary condition at $\tau_{*}=2 / 3: P=2 g / 3 \kappa(\kappa$ is the opacity) derived from the equation of hydrostatic equilibrium, which avoids the complication of the atmosphere modelisation.

From comparison of models 1,3 and 6,7 it is seen that the use of $\tau_{\star}=2 / 3$ for another law than Eddington's one does not introduce significant modification on the model. The simplified assumption SS gives a model close to the other ones not accurate enough for helioseismoly but sufficient for some problems of stellar evolution. The effect

TABLE I

\begin{tabular}{|c|c|c|c|c|c|c|}
\hline models & $T(\tau)$ & $\tau_{z}$ & $\tau_{b}$ & $\alpha$ & $Y$ & $R_{z c} / R_{\odot}$ \\
\hline 1 & 5750 & $2 / 3$ & 10 & 1.803 & 0.2744 & 0.721 \\
\hline 2 & 5750 & $2 / 3$ & 2 & 1.794 & 0.2744 & 0.721 \\
\hline 3 & 5750 & 0.548 & 10 & 1.794 & 0.2744 & 0.721 \\
\hline 4 & Edding & $2 / 3$ & 10 & 2.585 & 0.2744 & 0.721 \\
\hline 5 & Edding & $2 / 3$ & 2 & 1.678 & 0.2744 & 0.722 \\
\hline 6 & HSRA & $2 / 3$ & 2 & 1.899 & 0.2743 & 0.721 \\
\hline 7 & HSRA & 0.420 & 2 & 1.869 & 0.2743 & 0.721 \\
\hline 8 & SS & $2 / 3$ & $2 / 3$ & 1.798 & 0.2742 & 0.721 \\
\hline
\end{tabular}

of different treatments of the atmosphere on the sound speed is localized in the external layers. The differences of the p modes frequencies for degrees $l=0$ to 400 between models with same realistic $T(\tau)$ law (taking into account the convection) and different values of $\tau_{\star}$ and $\tau_{b}$ are less than $0.2 \mu \mathrm{Hz}$. The differences of the $\mathrm{p}$ modes frequencies between models with three different $T(\tau)$ laws (models 256 ) vary from zero for low frequency modes to $2 \mu \mathrm{Hz}$ for frequencies around $4 \mathrm{mHz}$.

In conclusion we have shown that the transfer equation reaches its asymptotic diffusion approximation at optical depths larger than ten. At such depths the energy is transported by convection and the $T(\tau)$ law needs to be derived from a model of atmosphere including convection and using consistent physics. However we find that the global properties and the oscillations frequencies of the solar calibrated models are not significantly sensitive to the optical thickness of the atmosphere provided it is larger than 2. The effect on the helioseismology of different $T(\tau)$ laws is rather small but not negligeable. More detailed analysis of the sensitivity of the solar model to the description of the atmosphere will be given in Morel et al. (1992).

\section{REFERENCES}

Ando H., Osaki Y. 1975, P.A.S.J., 27, 581

Kurucz R.L. 1992, private communication

Mihalas D. 1978, Stellar Atmosphere, 2d Ed. Freeman and Cie

Morel P., Provost J., Berthomieu G. 1990, Solar Physics 128, 7

Morel P., Van't Veer C., Berthomieu G., Cayrel R., Castelli F., Goupil M.J., Lebreton Y., Provost J. 1992, in preparation 